\title{
Natural convection adjacent to a sidewall with three fins in a differentially heated cavity F. $\mathrm{Xu}^{1} \quad$ J. C. Patterson ${ }^{2} \quad$ C. Lei $^{3}$
}

(Received 31 August 2006; revised 22 December 2007)

\begin{abstract}
Natural convection in a differentially heated cavity with three fins on a sidewall is numerically investigated. Due to the presence of the fins, separation of the thermal flows around the fins occurs, and the thermal flows separating from the fins oscillate. The oscillations of the thermal flows around the fins improve the convection flows adjacent to the finned sidewall, and the heat transfer through the finned sidewall is significantly enhanced (by up to $33 \%$ at the early stage). This flow configuration is of practical significance for many industrial systems.
\end{abstract}

\section{Contents}

See http://anziamj.austms.org.au/ojs/index.php/ANZIAMJ/article/view/133 for this article, (c) Austral. Mathematical Soc. 2007. Published December 31, 2007. ISSN $1446-8735$ 
2 Numerical procedures

C808

3 Time series of the thermal flows adjacent to the finned sidewall

C813

4 Flows adjacent to the finned sidewall and heat transfer C815

5 Conclusions

C817

References

C817

\section{Introduction}

Enhancement of the heat transfer through a differentially heated cavity is of significance in many industrial applications, and one method of enhancement is to place a fin on the sidewall [1]. Some of the fin parameters are critical for heat transfer enhancement, such as the dimension of the fin [7]. In addition, many researchers investigated natural convection in a cavity with multiple fins on the active sidewall for at least two decades. Those studies indicate that the flow patterns at the steady state are dependent on the Rayleigh number, the inclination angle of the cavity, the aspect ratio, and the dimension and separation interval of the fins. For a slender cavity with low Rayleigh numbers $\left(\mathrm{Ra}<10^{5}\right)$, a small cell may form in a 'micro-cavity', which consists of a sidewall and two neighboring fins [2,6]. Scozia and Frederick [6] indicated that, as the number of fins increases, the flow undergoes a transition over a number of distinct flow regimes, which have direct impact on the heat transfer through the sidewall.

For the conduction flow regime $\left(\mathrm{Ra}<10^{3}\right)$, if the fins are perfectly conducting the heat transfer increases with the increasing number of fins and the fin length due to the increasing heat transfer surface. For the convection regime, the dependence of heat transfer on the number and length of fins 
is complicated. As indicated by Yucel and Turkoglu [9], with an increasing number of fins the heat transfer first reaches a maximum and then approaches a constant, which is not affected by the number of fins. Lakhal et al. [3] also indicated that, if the cavity is inclined by $45^{\circ}$, the heat transfer generally decreases with increasing fin length.

Although studies on the effect of fins on laminar flows and heat transfer have extensively been reported, the above studies are mainly concerned with steady state flows in the range of low Rayleigh numbers $\left(\mathrm{Ra}<10^{7}\right)$. For higher Rayleigh numbers $\left(\mathrm{Ra}>10^{7}\right)$ at which natural convection becomes unsteady, the applicability of those results based on steady laminar flows evidently need to be re-examined. In this article natural convection in a differentially heated cavity with a Rayleigh number above $10^{9}$ is numerically simulated. We find that the transient thermal boundary layer flow arising from sudden heating approaches a periodic flow. Since the convection flow adjacent to the finned sidewall is enforced, the heat transfer through the sidewall is significantly enhanced. Comparisons of the flow and heat transfer between the configurations with three fins and without a fin are discussed.

\section{Numerical procedures}

A two dimensional domain, which is $H=0.24 \mathrm{~m}$ high by $L=1 \mathrm{~m}$ long, is considered, based on the experimental model [8]. Three fins of the same cross section of $20 \times 2 \mathrm{~mm}^{2}$ are placed on the hot sidewall with the same interval of $0.058 \mathrm{~m}$ between two neighbouring fins and the same distance of $0.059 \mathrm{~m}$ between the fin and horizontal wall, as seen in Figure 1. The working fluid is water. The two dimensional Navier-Stokes and energy equations with the Boussinesq approximation to be solved are

$$
\frac{\partial u}{\partial x}+\frac{\partial v}{\partial y}=0,
$$




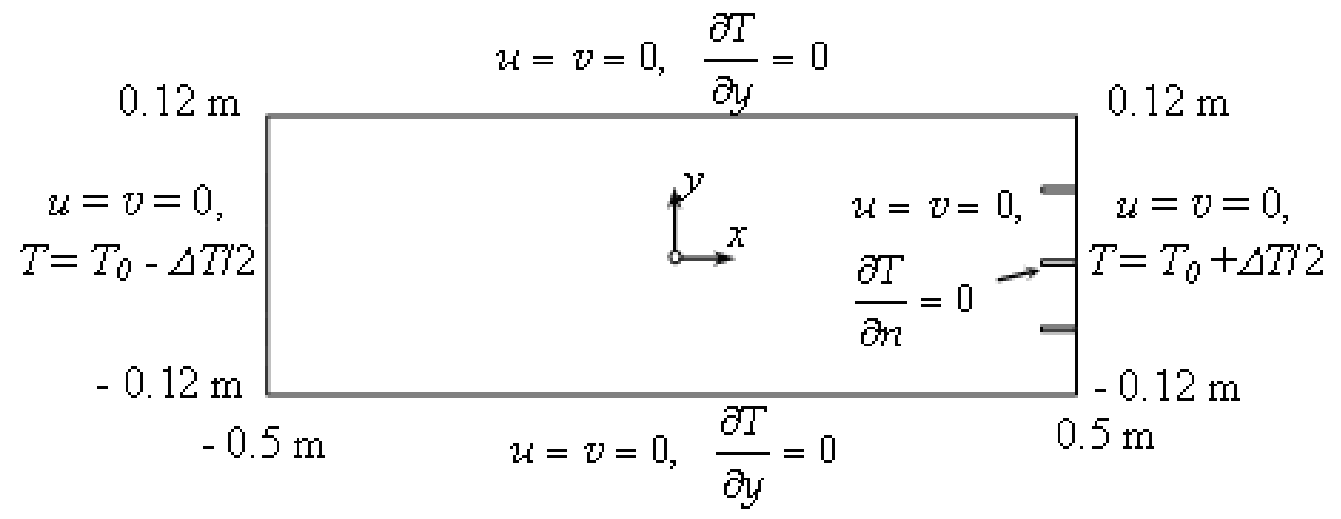

FiguRE 1: Schematic of the computational domain and boundary conditions.

$$
\begin{aligned}
& \frac{\partial u}{\partial t}+u \frac{\partial u}{\partial x}+v \frac{\partial u}{\partial y}=-\frac{1}{\rho} \frac{\partial p}{\partial x}+\nu\left(\frac{\partial^{2} u}{\partial x^{2}}+\frac{\partial^{2} u}{\partial y^{2}}\right), \\
& \frac{\partial v}{\partial t}+u \frac{\partial v}{\partial x}+v \frac{\partial v}{\partial y}=-\frac{1}{\rho} \frac{\partial p}{\partial y}+\nu\left(\frac{\partial^{2} v}{\partial x^{2}}+\frac{\partial^{2} v}{\partial y^{2}}\right)+g \beta\left(T-T_{0}\right), \\
& \frac{\partial T}{\partial t}+u \frac{\partial T}{\partial x}+v \frac{\partial T}{\partial y}=\kappa\left(\frac{\partial^{2} T}{\partial x^{2}}+\frac{\partial^{2} T}{\partial y^{2}}\right),
\end{aligned}
$$

where $T$ is the temperature, $T_{0}$ is the initial mean temperature, $p$ is the pressure, $u$ is the velocity in the $x$-direction, $v$ is the velocity in the $y$-direction, $g$ is the acceleration due to gravity, $\rho$ is the density, $\beta$ is the coefficient of thermal expansion, $\kappa$ is the thermal diffusivity, and $\nu$ is the kinematic viscosity. SI units are adopted for all quantities throughout the rest of the paper.

The boundary conditions are also shown in Figure 1 along with the coordinate system with the origin located at the center of the cavity. The top and bottom walls as well as surfaces of the fins are adiabatic, and a temperature difference is applied to the two sidewalls. Initially, the fluid in the cavity is motionless and isothermal $\left(T_{0}=295.55 \mathrm{~K}\right)$. The temperature difference between the two sidewalls is $16 \mathrm{~K}$. The corresponding Rayleigh number and 
Prandtl number are $3.77 \times 10^{9}$ and 6.64 , respectively:

$$
\mathrm{Ra}=\frac{g \beta \Delta T H^{3}}{\nu \kappa} \quad \text { and } \quad \operatorname{Pr}=\frac{\nu}{\kappa} .
$$

In order to estimate the degree of the enhancement of the heat transfer through the finned sidewall, we define a normalized difference of the heat transfer rate through the finned sidewall as

$$
q_{\text {diff }}=\frac{q_{\text {fins }}-q_{\text {nofin }}}{q_{\text {nofin }}},
$$

where $q_{\text {diff }}$ is a normalized difference of the heat transfer rate through the hot sidewall between the two cases with and without fins, $q_{\text {fins }}$ is the heat transfer rate through the entire sidewall with three fins, and $q_{\text {nofin }}$ is the heat transfer rate through the entire sidewall without fins. Furthermore, the local heat transfer coefficient and heat flux along the wall are

$$
h=\frac{q^{\prime}}{T_{w}-T_{0}} \quad \text { and } \quad q^{\prime}=k \frac{\partial T}{\partial n},
$$

where $T_{w}$ is the wall temperature, $q^{\prime}$ is the local heat flux of the wall, $h$ is the local heat transfer coefficient, $k$ is thermal conductivity, and $n$ is the direction normal to the wall surface.

The governing equations are solved using a finite volume SIMPLE algorithm [5]. All second derivatives and linear first derivatives are approximated by a second order center differenced scheme. The advection terms are discretized by a second order upwind scheme [7]. The time integration is discretized by a second order backward difference scheme. The discretized equations are iterated with under relaxation.

In order to accurately capture the features of the flows in the vicinity of the fins and wall boundaries, a non-uniform grid system is constructed with finer grids in the vicinity of the fins and wall boundaries. Similar to that used 

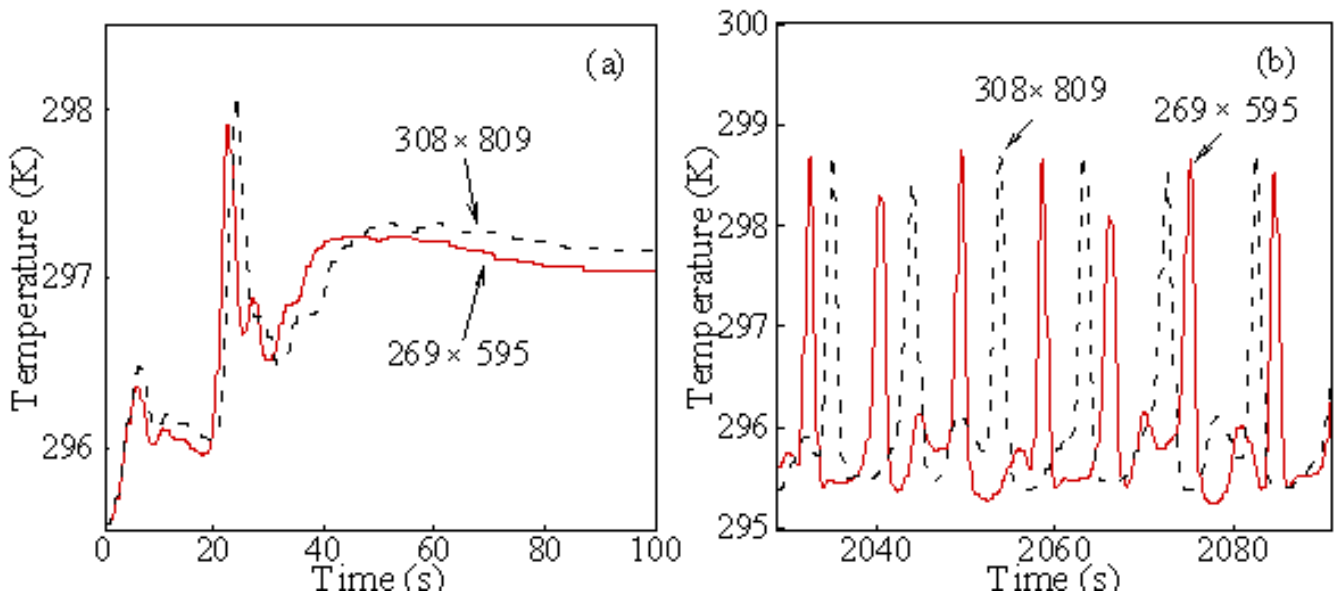

Figure 2: Time series of the temperatures at the point $(x=0.498 \mathrm{~m}, y=$ $-0.03 \mathrm{~m})$ calculated by different meshes at different times.

by $\mathrm{Xu}$ et al. [7], a $269 \times 595$ mesh is adopted. Furthermore, a finer mesh $(308 \times 809)$ is also tested, and the results of the mesh dependence test are shown in Figure 2, which plots the time series of the calculated temperatures at a typical point within the thermal boundary layer for the two meshes (solid and dashed lines). Clearly, the two meshes produce similar results in terms of the amplitude of temperature waves in both the early and transitional stages. However, details of temperature waves such as the phase and shape of temperature waves are sensitive to the mesh. Since the focus of this article is on the basic flow patterns and the transition process from sudden heating to a quasi-steady stage, the mesh of $269 \times 595$ is adopted in order to reduce the computational cost.

A time step of $0.1 \mathrm{~s}$ is adopted based on the previous studies by Patterson and Armfield [4] and Xu et al. [7]. Such a time step is sufficient to capture the features of transient flows, and the stability of the scheme is also guaranteed with the adoption of the non-uniform grids. 

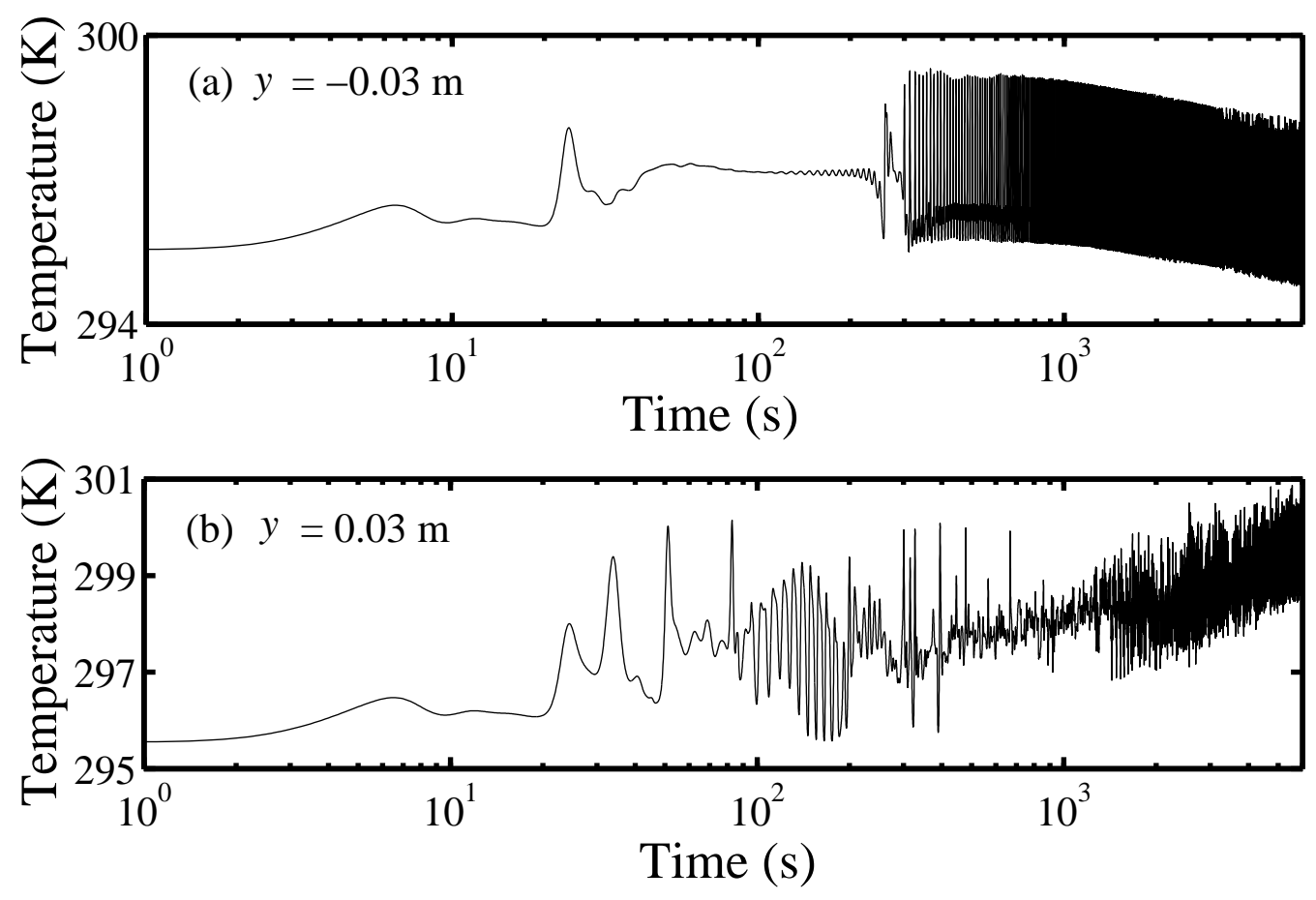

Figure 3: Time series of the temperatures at different heights $(x=0.498 \mathrm{~m})$. 

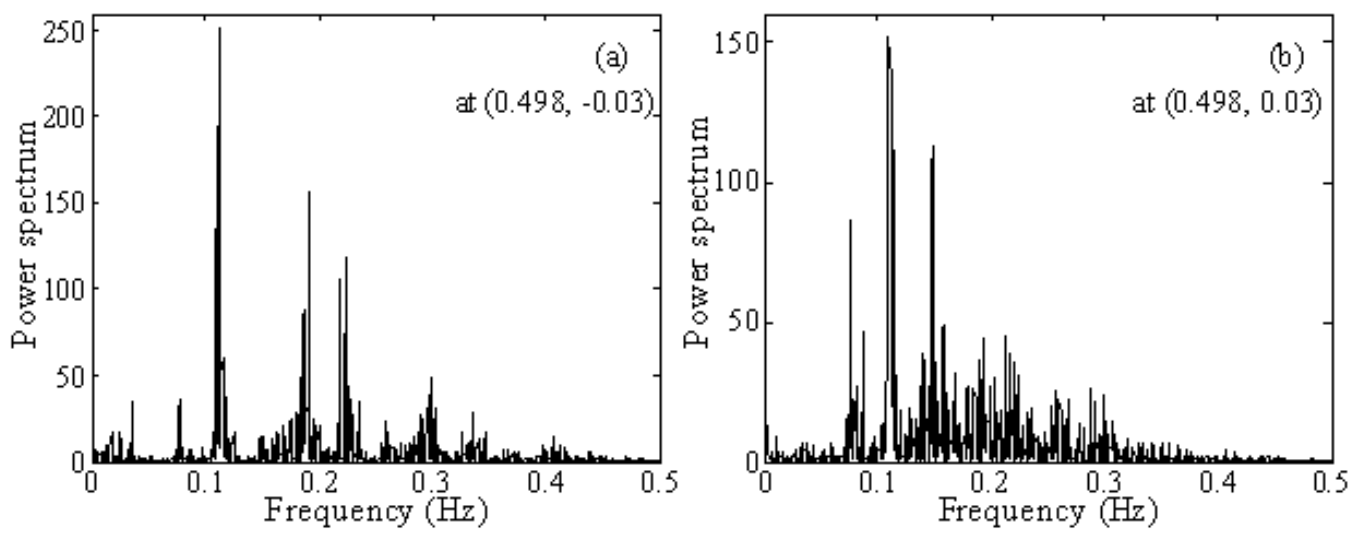

FiguRE 4: Spectra of the temperature time series at different points.

\section{Time series of the thermal flows adjacent to the finned sidewall}

For the purpose of observing the overall transition of the thermal flows in the vicinity of the finned sidewall from sudden heating to the quasi-steady stage, Figure 3 shows the time series of the temperatures obtained at different heights in the vertical thermal boundary layer. The temperature time series in Figure 3(a) is taken at the downstream side of the lowest fin $(x=0.498 \mathrm{~m}$, $y=-0.03 \mathrm{~m})$, and that in Figure 3(b) at the downstream side of the middle fin $(x=0.498 \mathrm{~m}, y=0.03 \mathrm{~m})$. Figure 3 shows that the lowest thin fin induces stronger downstream perturbations than the fin at the higher position by comparing the temperature waves in Figures 3(a) and (b) (note that both figures have a temperature scale of $6 \mathrm{~K}$ ). Eventually the thermal flows adjacent to the finned sidewall approach a periodic state (hereinafter referred to as quasi-steady state).

Figure 4 shows the corresponding spectra of the above temperature time series in the quasi-steady stage. Figure 4(a) indicates that the temperature 


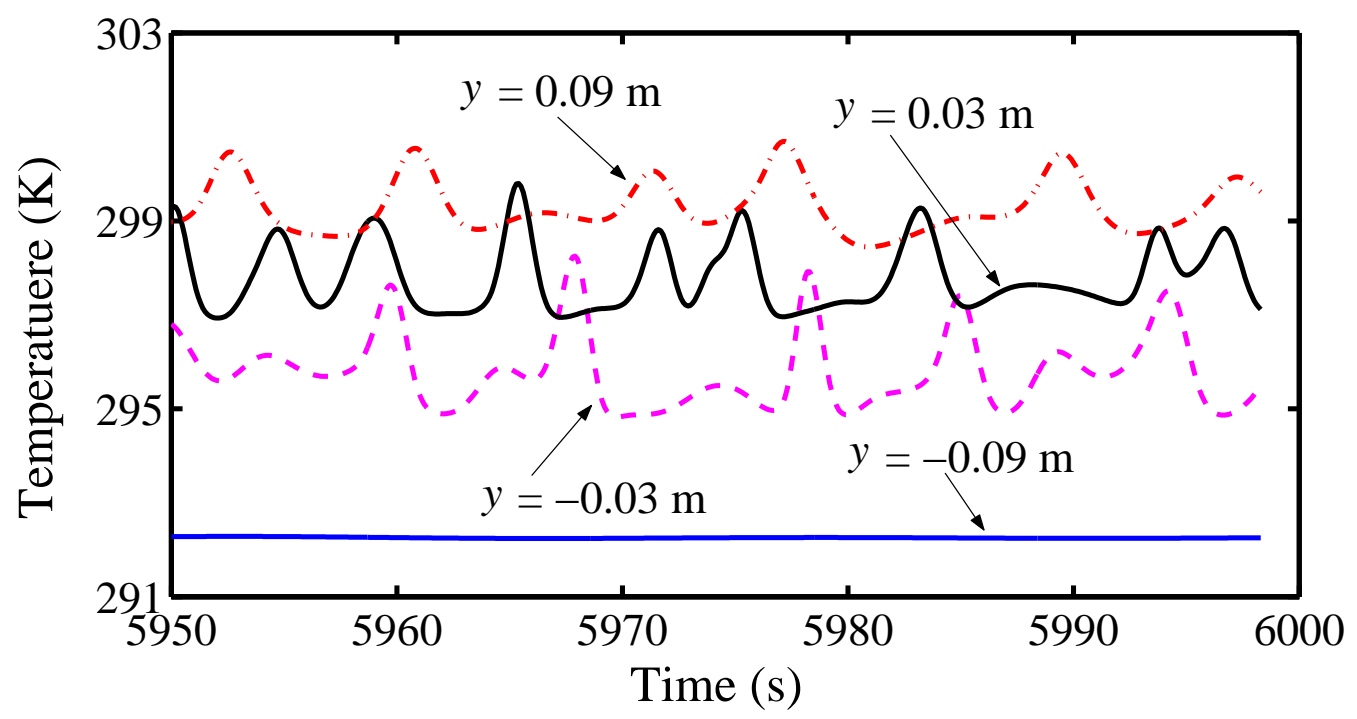

FiguRE 5: Time series of the temperatures at different heights $(x=0.498 \mathrm{~m})$.

waves induced by the lowest thin fin have a clear dominant frequency of $f=0.112 \mathrm{~Hz}$ with additional higher frequency modes. For the temperature waves induced by the thin fin at the higher position (Figure $4 \mathrm{~b}$ ), the dominant frequency is approximately at $f=0.11 \mathrm{~Hz}$, similar to that induced by the fin at the lower position, indicating that the perturbations from the upstream fin are carried downstream to the current location. However, the additional frequency modes at this location are evidently different from that at the lower position.

In order to observe further spatial evolution of the temperature waves in the thermal boundary layer, the time series of the temperatures obtained at different heights in the quasi-steady state are plotted in Figure 5. Since the temperature waves on the upstream side of the lowest fin are much weaker than those at the downstream side of the lowest fin, the wave feature of the temperature signal at the upstream side of the lowest fin cannot be shown clearly when the temperatures obtained at all the locations are plotted on 
the same scale (the temperature at the lowest position is shown as a straight line in Figure 5). Figure 5 also indicates that, apart from the upstream point of the lowest fin, the amplitude of the temperature waves decreases slightly as the monitoring position moves from upstream to downstream, which is different from those results induced by a single fin at the mid height of the sidewall [7].

\section{Flows adjacent to the finned sidewall and heat transfer}

Figure 6(a) shows the flows in the vicinity of the hot wall in the quasisteady state in which separations of the flows at the leeward side of the thin fins are clear, similar to that of the thermal flow around a single thin fin [7]. Oscillations of the thermal flow separating from the fins trigger the downstream instability of the thermal boundary layer flows, and result in the increasing convection flow on the downstream side of the fin. The effect of the increasing convection flow on the local heat transfer coefficient is plotted in Figure 6(b). The local heat transfer coefficient at the downstream sides of the fins is greater than that without the fins (the dashed line), implying that the local heat transfer through the hot finned sidewall is enhanced at this time.

For the purpose of quantitatively estimating the degree of the enhancement of the heat transfer through the finned sidewall, Figure 7 plots the normalized difference of the heat transfer rate through the hot wall against time (in which the dashed lines are the scale grid lines), and indicates that the heat transfer is enhanced by as much as $33 \%$ in the initial stage. 

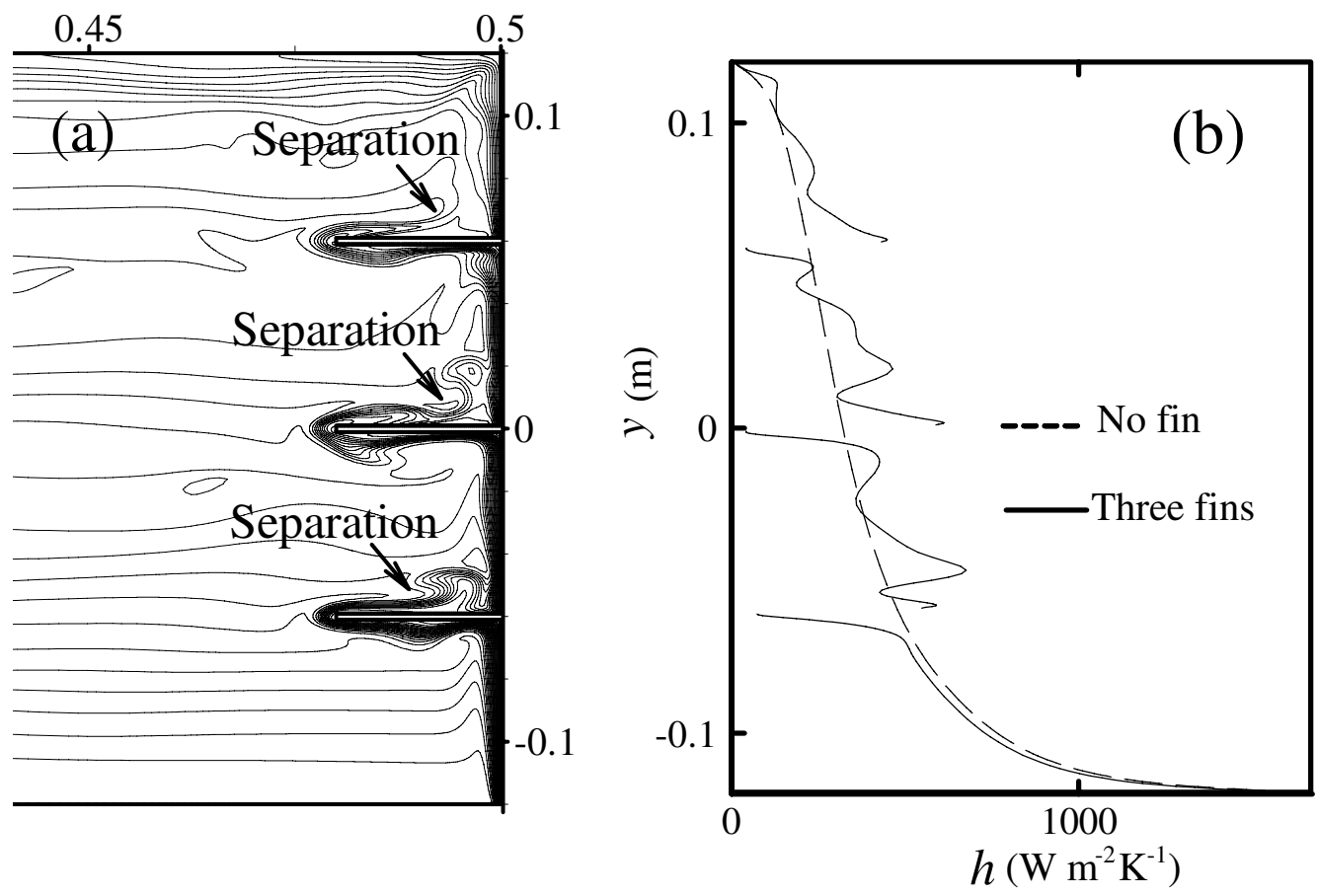

Figure 6: Flow and heat transfer at $t=6000 \mathrm{~s}$ : (a) Isotherms (from 287.55 to $303.55 \mathrm{~K}$ with the interval of $0.4 \mathrm{~K}$ ); (b) Heat transfer coefficient.

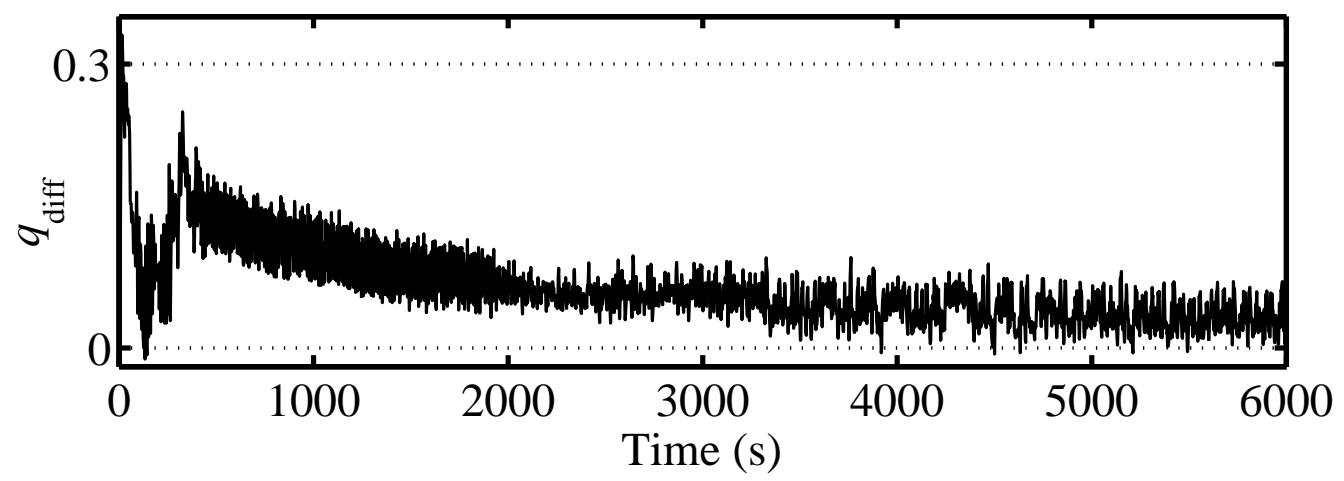

FiguRE 7: $q_{\text {diff }}$ versus time. 


\section{Conclusions}

Natural convection in a differentially heated cavity with three fins placed on the hot wall has been numerically simulated. The results are compared with the case without a fin. The thermal flows around the fins separate from the fins and oscillate. The oscillations of the thermal flows around the fins improve the convection flow on the downstream side of the fins, and the local heat transfer on the downstream side of the fins is enhanced. As a result, the total heat transfer through the finned sidewall is significantly enhanced, as shown in Figure 7. Further investigations of the effect of the fin on the flows and heat transfer, which consider different geometries and sizes of the fin, are currently underway.

Acknowledgement: The financial support of the Australian Research Council is gratefully acknowledged.

\section{References}

[1] E. Bilgen, Natural convection in cavities with a thin fin on the hot wall, Int. J. Heat Mass Transfer, 48, 3493-3505, 2005. doi:10.1016/j.ijheatmasstransfer.2005.03.016 C807

[2] G. N. Facas, Natural convection in a cavity with fins attached to both vertical walls, J. Thermophys. Heat Transfer, 7, 555-560, 1993. C807

[3] E. K. Lakhal, M. Hasnaoui, E. Bilgen, and P. Vasseur, Natural convectin in inclined rectangular enclosures with perfectly conducting fins attached on the heated wall, Heat Mass Transfer, 32, 365-373, 1997. C808 
[4] J. C. Patterson and S. W. Armfield, Transient features of natural convection in a cavity, J. Fluid Mech., 219, 469-497, 1990. doi:10.1017/S0022112090003032 C811

[5] S. V. Patankar, Numerical heat transfer and fluid flow, Hemisphere Publishing Corp., 1980 C810

[6] R. Scozia and R. L. Frederick, Natural convection in slender cavities with multiple fins attached on an active wall, Numer. Heat Transfer Part A 20, 127-158, 1991. C807

[7] F. Xu, J. C. Patterson and C. Lei, Oscillations induced by a thin fin on the sidewall of a differentially heated cavity, Proc. 13th International Heat Transfer Conference, Sydney, 2006. C807, C810, C811, C815

[8] F. Xu, J. C. Patterson and C. Lei, Experimental observations of the thermal flow around a square obstruction on a vertical wall in a differentially heated cavity, Exp. Fluids, 40, 364-371, 2006. doi:10.1007/s00348-005-0073-x C808

[9] N. Yucel and H. Turkoglu, Numerical analysis of laminar natural convection in enclosures with fins attached to an active wall, Heat and Mass Transfer, 33, 307-314, 1998. C808 


\section{Author addresses}

1. F. Xu, School of Engineering, James Cook University, Townsville, Australia.

mailto:feng.xu@jcu.edu. au

2. J. C. Patterson, School of Engineering, James Cook University, Townsville, Australia.

3. C. Lei, School of Engineering, James Cook University, Townsville, Australia. 